\title{
Quantitative phase imaging using grating-based quadrature phase interferometer
}

Jigang Wu, Zahid Yaqoob, Xin Heng, Xiquan Cui, Changhuei Yang

Jigang Wu, Zahid Yaqoob, Xin Heng, Xiquan Cui, Changhuei Yang, "Quantitative phase imaging using grating-based quadrature phase interferometer," Proc. SPIE 6443, Three-Dimensional and Multidimensional Microscopy: Image Acquisition and Processing XIV, 64430J (14 February 2007); doi: 10.1117/12.701694

SPIE. Event: SPIE BiOS, 2007, San Jose, California, United States 


\title{
Quantitative phase imaging using grating-based quadrature phase interferometer
}

\author{
Jigang $\mathrm{Wu}^{*}$, Zahid Yaqoob, Xin Heng, Xiquan Cui, Changhuei Yang \\ Biophotonics Laboratory, Department of Electrical Engineering \\ California Institute of Technology, Pasadena, CA, 91125
}

\begin{abstract}
In this paper, we report the use of holographic gratings, which act as the free-space equivalent of the $3 \times 3$ fiber-optic coupler, to perform full field phase imaging. By recording two harmonically-related gratings in the same holographic plate, we are able to obtain nontrivial phase shift between different output ports of the gratings-based Mach-Zehnder interferometer. The phase difference can be adjusted by changing the relative phase of the recording beams when recording the hologram. We have built a Mach-Zehnder interferometer using harmonically-related holographic gratings with 600 and 1200 lines $/ \mathrm{mm}$ spacing. Two CCD cameras at the output ports of the gratings-based Mach-Zehnder interferometer are used to record the full-field quadrature interferograms, which are subsequently processed to reconstruct the phase image. The imaging system has $\sim 12 \mathrm{X}$ magnification with $\sim 420 \mu \mathrm{mx} 315 \mu \mathrm{m}$ field-of-view. To demonstrate the capability of our system, we have successfully performed phase imaging of a pure phase object and a paramecium caudatum.
\end{abstract}

Keywords: Diffraction gratings, quadrature phase interferometry, medical and biological imaging

\section{INTRODUCTION}

Phase contrast ${ }^{1,2}$ and Nomarski microscopy ${ }^{3}$ are widely used to image transparent objects such as living cells. However, these techniques cannot directly provide quantitative phase information of the sample. In the past years, many quantitative phase techniques have been developed, which include interferometric techniques such as phase-shifting interferometry ${ }^{4}$, polarization based phase microscopy ${ }^{5}$, digital holography ${ }^{6}$, Fourier phase microscopy ${ }^{7}$ and Hilbert phase microscopy, etc. ${ }^{8}$, and noninterferometric techniques ${ }^{9,10} .3 \times 3$ fiber-optic couplers, which feature non-trivial phase shifts between the different output ports of the coupler, have been shown to be useful for quantitative phase measurements ${ }^{11}$. However, the $3 \times 3$ couplers technique cannot be used to perform full field phase imaging.

Recently, we reported that a harmonically-related diffraction grating pair can introduce nontrivial phase shift in the outputs of the interferometer ${ }^{12}$. In comparison, a single shallow grating used as a beam combiner in the interferometer can only generate $\pi$ phase shift. In this paper, we show that a harmonically-related diffraction grating pair can be recorded into the same holographic plate. The holographic grating was then used as a beam combiner in our MachZehnder interferometer system and phase imaging of transparent objects was performed.

\section{THEORY}

As derived in ref. 12, for a single shallow grating, the relative phase shift of the $m^{\text {th }}$ diffracted order with respect to the zeroth order is given by:

$$
\phi\left(x_{0}\right)=\left\{\begin{array}{cl}
m\left[\frac{2 \pi x_{0}}{\Lambda}+\frac{\pi}{2}\right], & m \geq 1 \\
|m|\left[-\frac{2 \pi x_{0}}{\Lambda}+\frac{\pi}{2}\right], & m \leq-1
\end{array}\right.
$$

\footnotetext{
*jigang@caltech.edu; phone 16263954711
}

Three-Dimensional and Multidimensional Microscopy: Image Acquisition and Processing XIV,

edited by Jose-Angel Conchello, Carol J. Cogswell, Tony Wilson, Proc. of SPIE Vol. 6443, 64430J, (2007)

1605-7422/07/\$18 - doi: 10.1117/12.701694 
where $x_{0}$ is the displacement of the grating from some predefined origin (the coordinate system is shown in fig.1), and $\Lambda$ is the period of the grating. According to the phase shift equation (1), a $\pi$ phase shift will be obtained if a single shallow grating is used as a beam combiner in an interferometer ${ }^{12}$, as shown in fig.1 (a). Here we define G1 and G2 as harmonically related single gratings with $\Lambda_{1}=2 \Lambda_{2}$, and G1G2 is a combined grating pair of G1 and G2 gratings. Instead of a single grating G1, if a grating pair G1G2 is used, nontrivial phase shift can be obtained, as shown in fig.1 (b). In fig. $1, x_{1}, x_{2}$ are the displacements of the single grating G1, G2 with respect to the origin; $\phi_{m, G n}$ is the phase shift of the $m^{\text {th }}$ diffracted order with respect to the zeroth order caused by the grating $\mathrm{Gn}(\mathrm{n}=1,2) ; \phi_{k, n}(\mathrm{k}=\mathrm{r}, \mathrm{s} ; \mathrm{n}=1,2)$ is the phase shift of reference beam $(\mathrm{k}=\mathrm{r})$ or sample beam $(\mathrm{k}=\mathrm{s})$ after diffracted by the grating in the output port $n ; \Delta \phi_{n}$ $(\mathrm{n}=1,2)$ is the additional phase of the interference term in the output port $n$ caused by the grating diffraction; $\Delta \phi$ is the phase shift between the two output ports.

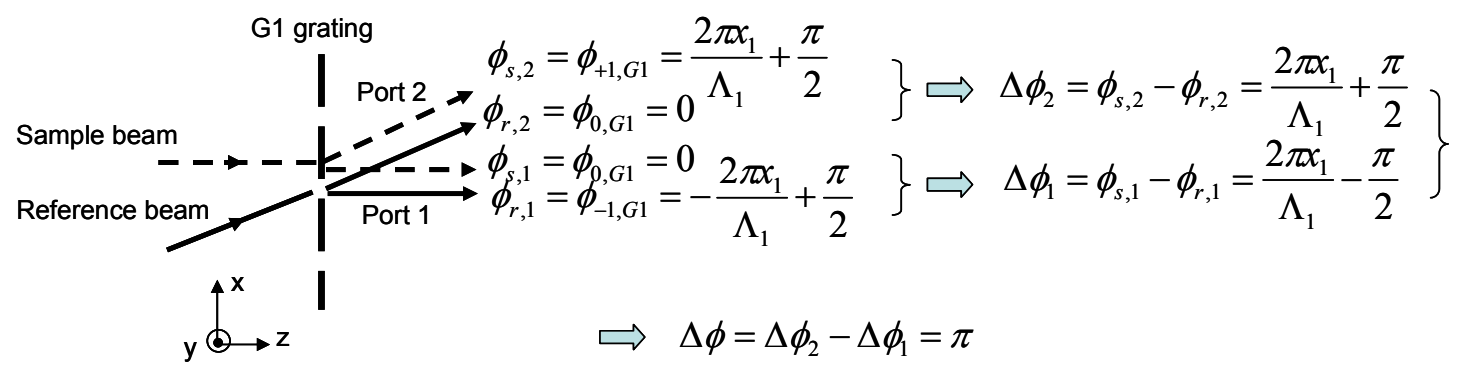

(a)

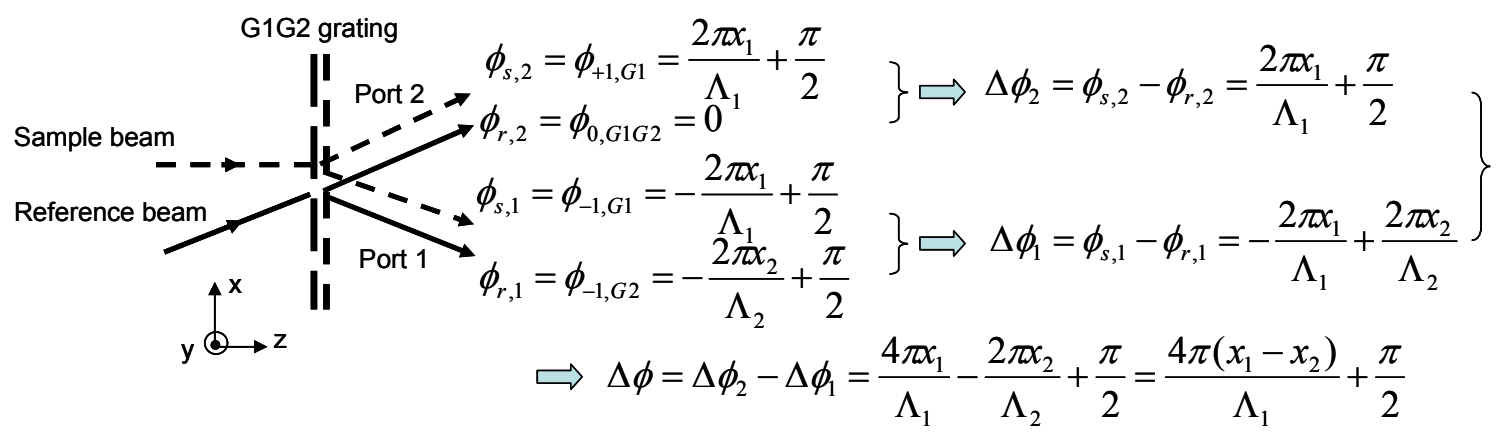

(b)

Fig. 1. principles of getting nontrivial phase by using G1G2 grating. (a) a single G1 grating can only introduce $\pi$ phase shift. (b) a harmonically related G1G2 grating can introduce nontrivial phase shift.

According to fig.1 (b), the phase shift in the output ports of a G1G2 grating is

$$
\Delta \phi=\frac{4 \pi\left(x_{1}-x_{2}\right)}{\Lambda_{1}}+\frac{\pi}{2}
$$

$x_{1}-x_{2}$ can be adjusted in the recording process to get nontrivial phase shift. Since $x_{1}-x_{2}$ is fixed after the holographic grating is developed, the phase shift $\Delta \phi$ is uniform across the grating surface. Note that the above result depends on the harmonic relation of the two gratings, so if the grating pairs are not harmonically related, we should expect to see different phase shift $\Delta \phi$ across the grating surface. 
In the following we will derive the equations for obtaining phase and intensity information by using a harmonically related G1G2 grating as a beam combiner. When the G1G2 grating is used as a beam combiner in an interferometer setup, as shown in fig. 1 (b), the detected electric field in the output port $1 E_{l}$ is the superposition of the -1 order diffraction of the sample beam $E_{s}$ by G1 grating and the -1 order diffraction of the reference beam Er by G2 grating. Here we neglect the -2 order diffraction of the reference beam $E_{r}$ by G1 grating as the diffraction is very weak. Similarly, in the output port $2, E_{2}$ is the superposition of the +1 order diffraction of the sample beam $E_{s}$ by G1 grating and the 0 order diffraction of the reference beam $E_{r}$ by G1G2 grating.

Using subscript 1, 2 to denote G1 and G2 gratings, respectively, the electric fields in the output ports of the G1G2 grating can be written as

$$
\begin{aligned}
& E_{1}=\eta_{-1, G 1} E_{s} \exp \left[i\left(\psi_{s}+\phi_{-1, G 1}\right)\right]+\eta_{-1, G 2} E_{r} \exp \left[i\left(\psi_{r}+\phi_{-1, G 2}\right)\right] \\
& E_{2}=\eta_{+1, G 1} E_{s} \exp \left[i\left(\psi_{s}+\phi_{+1, G 1}\right)\right]+\eta_{0, G 1 G 2} E_{r} \exp \left(i \psi_{r}\right)
\end{aligned}
$$

where $\psi_{r}, \psi_{s}$ are the phase of the electric field including the phase caused by propagation of the light and other additional phase; $\phi_{m, G n}$ has been defined above; and $\eta_{m, G n}$ is the electric field diffraction efficiency of the $m^{\text {th }}$ diffracted order of the grating $\mathrm{Gn}(\mathrm{n}=1,2)$. Here we omit the time dependent term $\exp (-i \omega t)$. Thus the detected powers in the output ports are

$$
\begin{aligned}
& P_{1}=\left\langle E_{1}^{*} E_{1}\right\rangle=\left(\eta_{-1, G 2} E_{r}\right)^{2}+\left(\eta_{-1, G 1} E_{s}\right)^{2}+2 \eta_{-1, G 1} \eta_{-1, G 2} E_{r} E_{s} \cos \left(\psi_{s}+\phi_{-1, G 1}-\psi_{r}-\phi_{-1, G 2}\right) \\
& P_{2}=\left\langle E_{2}^{*} E_{2}\right\rangle=\left(\eta_{0, G 1 G 2} E_{r}\right)^{2}+\left(\eta_{+1, G 1} E_{s}\right)^{2}+2 \eta_{0, G 1 G 2} \eta_{+1, G 1} E_{r} E_{s} \cos \left(\psi_{s}+\phi_{+1, G 1}-\psi_{r}\right)
\end{aligned}
$$

In experiment, usually the reference beam power will be much stronger than the sample beam power, thus equation (4) and (5) can be written as

$$
\begin{aligned}
& P_{1} \approx P_{r 1}+2 \sqrt{P_{r 1} P_{s 1}} \cos (\Delta \psi) \\
& P_{2} \approx P_{r 2}+2 \eta \sqrt{P_{r 2} P_{s 1}} \cos (\Delta \psi+\Delta \phi)
\end{aligned}
$$

where $P_{r 1}=\left(\eta_{-1, G 2} E_{r}\right)^{2}, P_{r 2}=\left(\eta_{0, G 1 G 2} E_{r}\right)^{2}$ are the detected reference powers in the output ports 1 and 2 , respectively; $P_{s 1}=\left(\eta_{-1, G 1} E_{s}\right)^{2}$ is the detected sample power in the output port $1 ; \eta=\eta_{+1, G 1} / \eta_{-1, G 1}$; $\Delta \psi=\psi_{s}-\psi_{r}$; and $\Delta \phi$ is given by eq. (2). In the experiment, we can measure $P_{1}, P_{2}, P_{r 1}, P_{r 2}, \Delta \phi$, and $\eta$. Solving equation (7) and (8) will give us the phase of the sample (relative to the reference) and the intensity information as well:

$$
\Delta \psi=\tan ^{-1} \frac{s_{1} \cos \Delta \phi-s_{2}}{s_{1} \sin \Delta \phi}, P_{s 1}=\left(\frac{s_{1}}{\cos (\Delta \psi)}\right)^{2}
$$

where

$$
s_{1}=\frac{P_{1}-P_{r 1}}{2 \sqrt{P_{r 1}}}, s_{2}=\frac{P_{2}-P_{r 2}}{2 \eta \sqrt{P_{r 2}}}
$$

\section{EXPERIMENT SETUP}

The experiment setup for recording the holographic grating is shown in fig.2. The laser is coupled into a single mode fiber (SMF) by an objective lens. The output from the SMF is collimated by lens 1 and split into two beam by 
beam splitter 1. Beam splitter 2 further splits the beam so that we have a total of three beams split from the same laser source. Beam splitter 3 is added to make sure the powers of the three beams are the same. The three beams are aligned using a standard commercially available grating with 600 lines $/ \mathrm{mm}$ so that the recorded gratings will be 600 lines $/ \mathrm{mm}$ and 1200 lines $/ \mathrm{mm}$. Then the standard grating is replaced by a holographic plate. The three beams incident on the holographic plate interfere with one another and generate two G1 gratings and one G2 grating. The two G1 gratings can be treated as a combined G1 grating, thus we have the G1G2 grating after developing and bleaching the holographic plate.

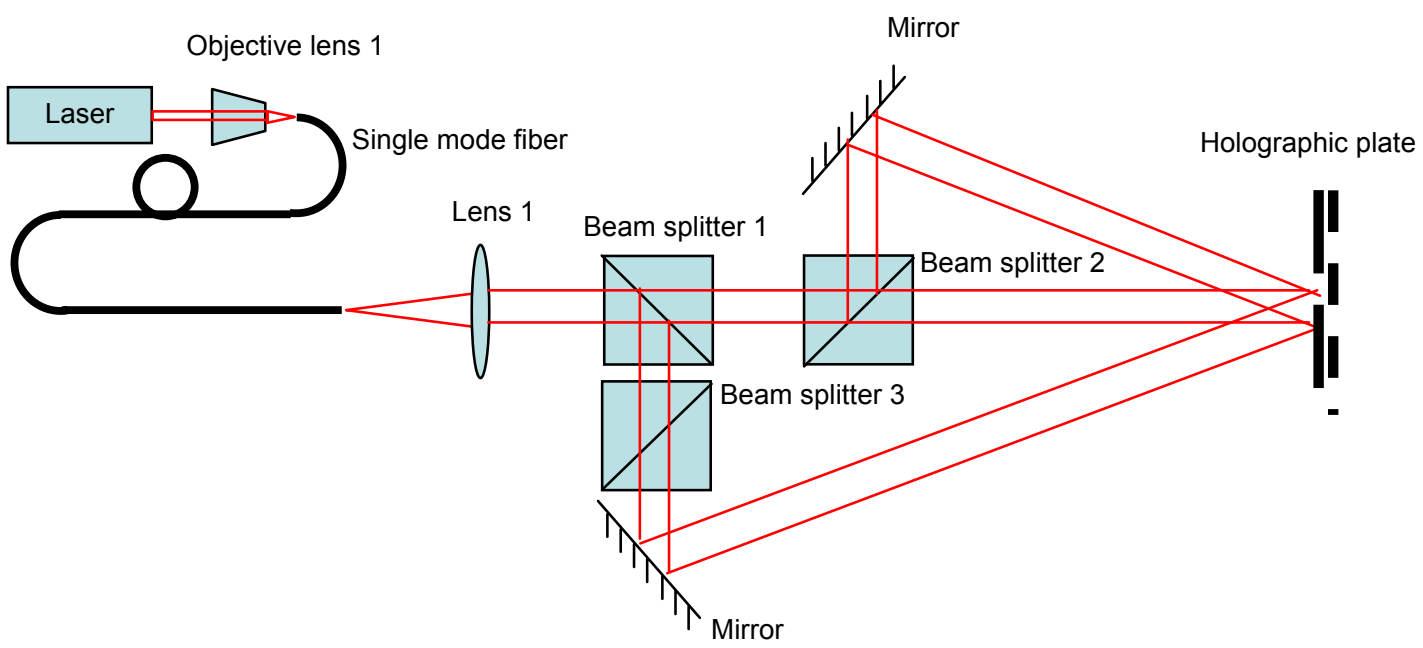

Fig. 2. Experiment setup for recording the holographic grating.

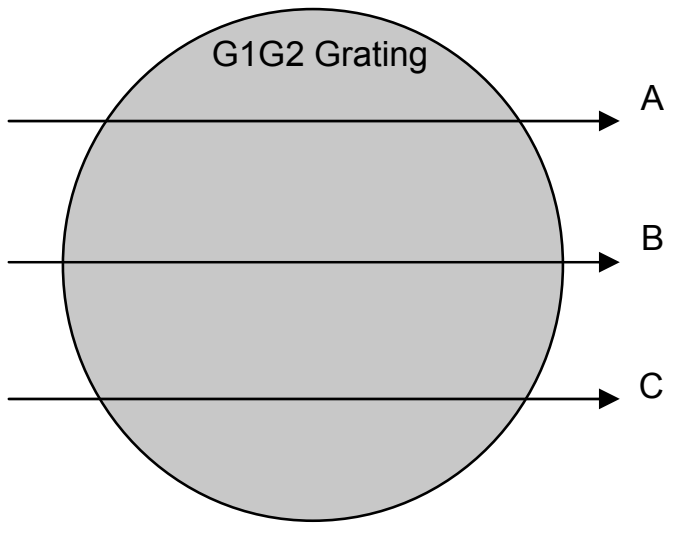

(a)

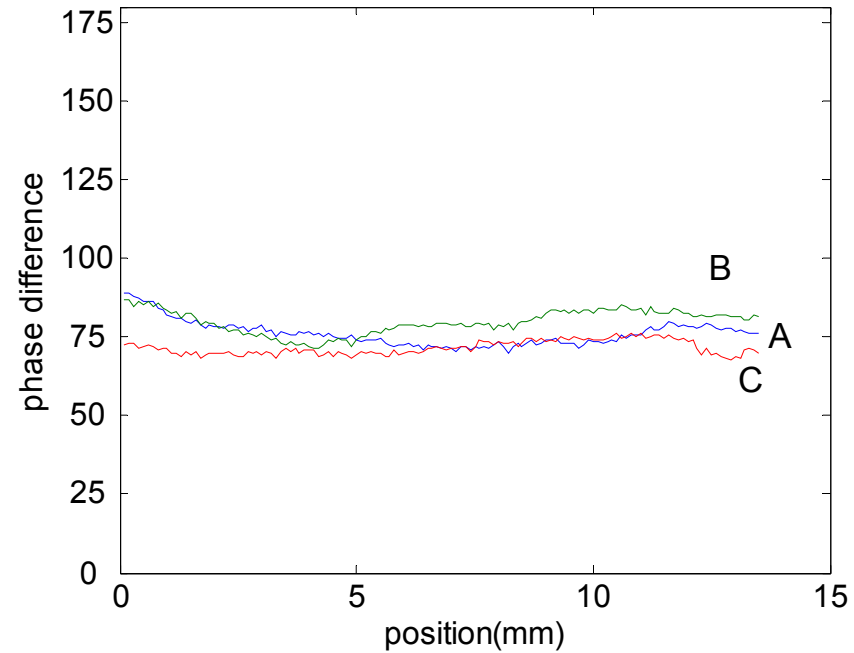

(b)

Fig. 3. Phase shift across the G1G2 grating measured in three different line locations. (a) Three different line locations indicated by A, B, and C. (b) The corresponding phase shifts. 
The G1G2 grating is then calibrated by a simple Mach-Zehnder interferometer to determine the phase shift $\Delta \phi$ between the two output ports. The grating is mounted on a translation stage so that the phase shift across the grating surface can be measured. The phase shift measured in three different line locations indicated in fig.3 (a) are shown in fig. 3 (b). The deviation is less than $+/-10$ degree and the average phase shift is about 75 degree, which we will use as $\Delta \phi$ in the experiment data processing.

The experimental setup for phase imaging is shown in fig. 4. The laser is focused by the objective lens 1 and the pinhole acts as a spatial filter. Then the laser is collimated by lens 1 and split by the beam splitter. In the sample arm, the objective lens 2 and lens 2 make up a microscope system and image the sample onto the CCD. The G1G2 grating acts as a beam combiner and combine the reference beam and the sample beam just as shown in fig.1 (b). In the experiment, the reference beam is aligned so that a "bull's eye" pattern can be seen if no sample is present. The CCDs are aligned to make sure the acquired images overlapped with each other. Then the corresponding pixels from CCD1 and CCD2 will detect the laser power satisfying equation (7)-(10).

Before acquiring phase image, the grating efficiency $\eta$ and the reference beam powers $P_{r l}, P_{r 2}$ are measured. So during the imaging, we need only to measure P1 and P2. The phase and intensity information can be derived according to equation (9) and (10) for each pixel.

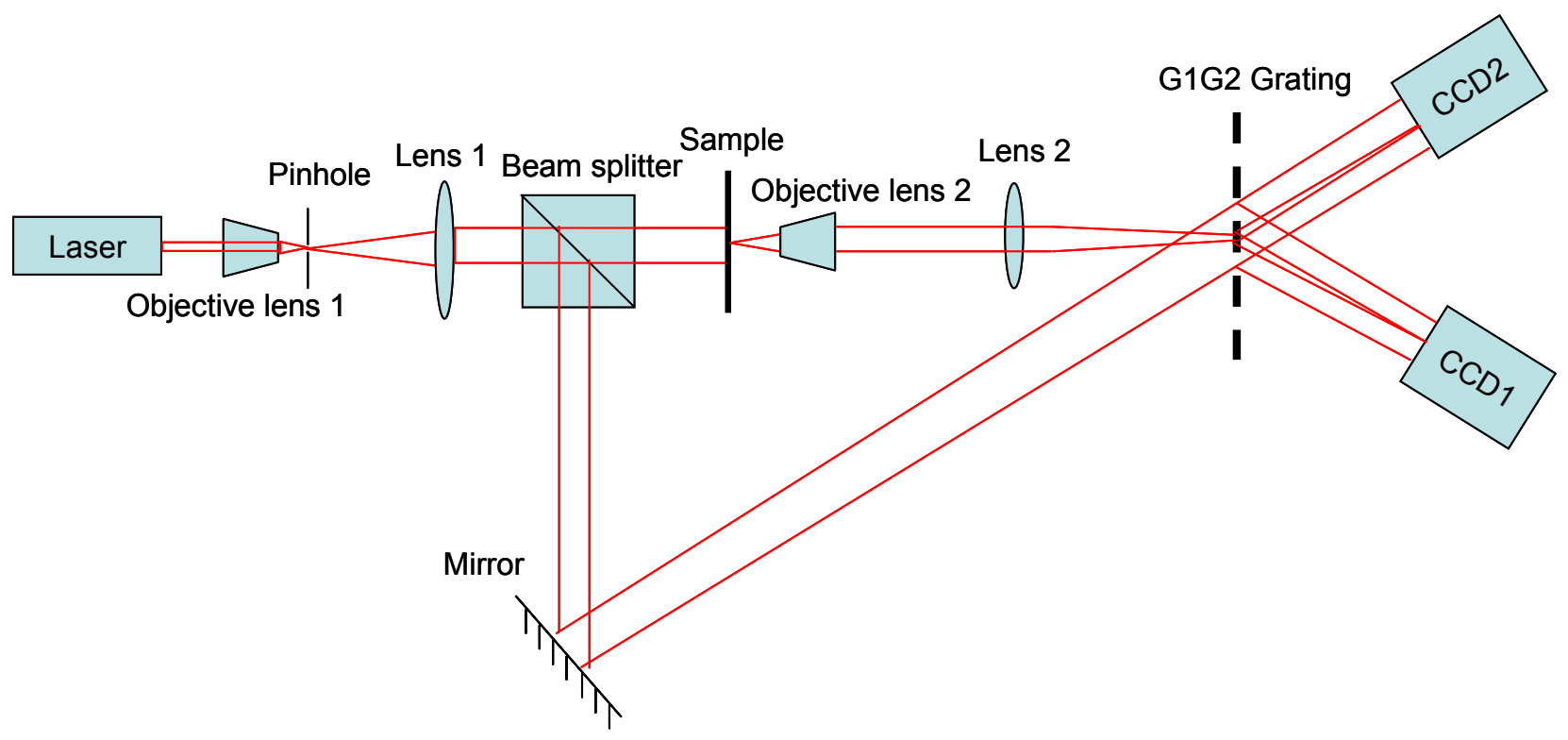

Fig. 4. Experiment setup for phase imaging.

\section{IMAGING RESULT}

To verify our system, a standard phase object is fabricated for imaging. An ITO glass is spin coated with $100 \mathrm{~nm}$ PMMA and two bars are written on the PMMA by E beam lithography. Both the width of the bar and the separation between them are $80 \mu \mathrm{m}$.

The imaging result is shown in fig. 5. In the intensity image fig. 5 (a), there is no contrast inside and outside the bars and only the edge of the bar is barely visible. In the phase image fig. 5 (b), however, the contrast is much better. The 3-D rendering of the phase image is shown in fig. 5 (c). Quantitative depth of the measurement is shown in fig. 5 (d), compared with the AFM measurement.

The phase difference of the regions inside and outside the bars can be written as: 


$$
\Delta \theta=(n-1) \frac{2 \pi d}{\lambda}
$$

where $n=1.4962$ is the refractive index of PMMA; $d$ is the depth of the bars; and $\lambda=0.633 \mathrm{~mm}$ is the wavelength of the He-Ne laser source. Thus $d$ can be derived by the measuring $\Delta \theta$. We can see from fig. 5 (d) that the error of $d$ is $\sim 50 \mathrm{~nm}$, and the corresponding phase error is $\sim 14^{\circ}$.

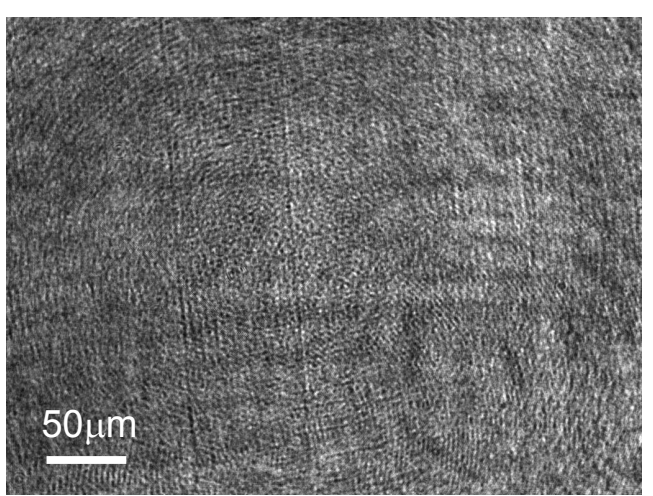

(a)



(c)

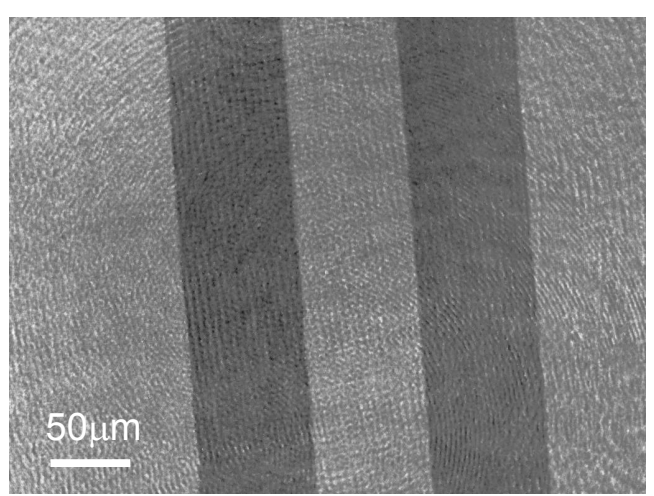

(b)

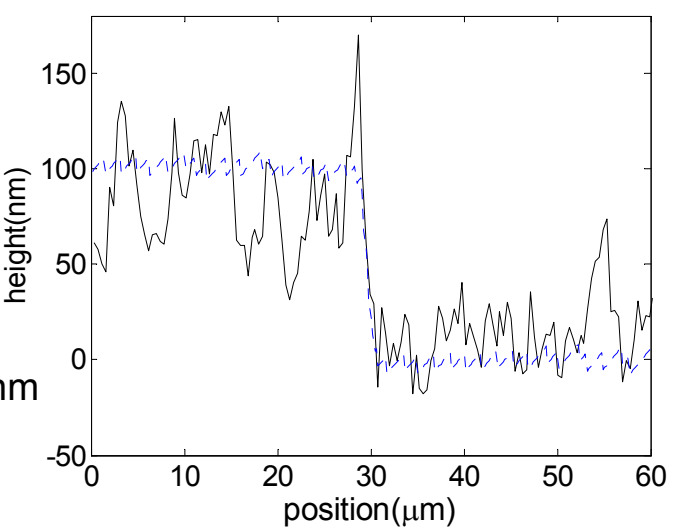

(d)

Fig. 5. Imaging a standard phase object. (a) Intensity image; (b) phase image; (c) 3-D rendering of the phase image; (d) quantitative phase measurement compared with AFM.

Fig. 6 shows the acquired phase image of a paramecium caudatum. The oral groove, cytostome and cilia can be discerned in the image. 




(a)

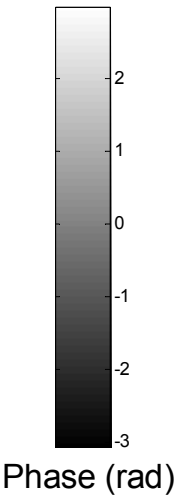

Phase (rad)

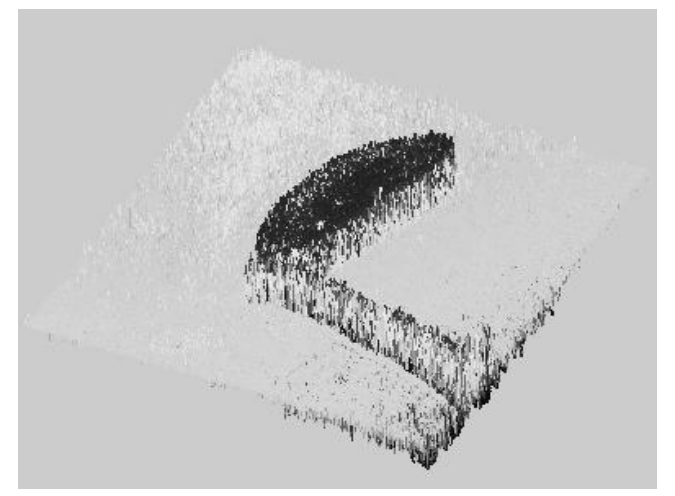

(b)

Fig. 6. Image of a paramecium caudatum. (a) phase image, the orgal groove, cytostome and cilia can be discern; (b) 3-D rendering of the phase image;

\section{CONCLUSION}

In conclusion, we have shown that a harmonically grating pair can be used to acquire both quantitative phase and intensity information of the sample. Compared with Hilbert phase microscopy and digital holography, data processing is relatively easy. In addition, high spatial frequency fringes, which required high resolution CCD camera, are not required. This promising phase imaging technique may be potentially useful in live cell imaging and may be used to study cell dynamics.

\section{REFERENCES}

1. F. Zernike, "Phase contrast, a new method for the microscopic observation of transparent objects", Physica 9, 686698 (1942).

2. F. Zernike, "Phase contrast, a new method for the microscopic observation of transparent objects Part II", Physica 9, 974-986 (1942).

3. M. G. Nomarski, "Microinterferometre Differentiel a Ondes Polarisees", Journal De Physique Et Le Radium 16, S9-S13 (1955).

4. K. Creath, in Progess in Optics, Vol. XXVI, E. Wolf, ed. (Elsevier, Amsterdam, 1998), pp349-393.

5. D. Do. Hogenboom, et al., "Three-dimensional images generated by quadrature interferometry", Optics Letters 23 (10), 783-785 (1998)

6. P. Marquet, et al., "Digital holographic microscopy: a noninvasive contrast imaging technique allowing quantitative visualization of living cells with subwavelength axial accuracy", Optics Letters 30 (5), 468-470 (2005).

7. G. Popescu, et al., "Fourier phase microscopy for investigation of biological structures and dynamics", Optics Letters 29 (21), 2503-2505 (2004).

8. T. Ikeda, et al., "Hilbert phase microscopy for investigating fast dynamics in transparent systems", Optics Letters 30 (10), 1165-1167 (2005).

9. A. Barty, et al., "Quantitative optical phase microscopy", Optics Letters 23 (11), 817-819 (1998).

10. D. Paganin and K. A. Nugent, " Noninterferometric phase imaging with partially coherent light", Physical Review Letters, 80 (12), 2586-2589 (1998). 
11. M. Choma, et al., "Instantaneous quadrature low-coherence interferometry with $3 \times 3$ fiber-optic couplers", Optics Letters 28(22), 2162-2164 (2003)

12. Z. Yaqoob, et al., "Harmonically-related diffraction gratings-based interferometer for quadrature phase measurements", Optics Express 14 (18), 8127-8137 (2006). 